\title{
Defective Neutrophil Function in Patients with Sepsis Is Mostly Restored by ex vivo Ascorbate Incubation
}

\author{
Kritsanawan Sae-khow' \\ Sasipha Tachaboon ${ }^{2}$ \\ Helen L Wright ${ }^{3}$ \\ Steven W Edwards ${ }^{4}$ \\ Nattachai Srisawat ${ }^{2}$ \\ Asada Leelahavanichkul' \\ Direkrit Chiewchengchol' \\ 'Translational Research in Inflammation \\ and Immunology Research Unit, Faculty \\ of Medicine, Chulalongkorn University, \\ Bangkok, Thailand; ${ }^{2}$ Excellence Center \\ for Critical Care Nephrology, King \\ Chulalongkorn Memorial Hospital, Thai \\ Red Cross Society, Bangkok, Thailand; \\ ${ }^{3}$ Institute of Ageing and Chronic Disease, \\ University of Liverpool, Liverpool, UK; \\ ${ }^{4}$ Institute of Integrative Biology, \\ University of Liverpool, Liverpool, UK
}

Correspondence: Direkrit

Chiewchengchol

Email cdirekrit@live.com
This article was published in the following Dove Press journal: Journal of Inflammation Research

Background: Neutrophil function is essential for effective defence against bacterial infections but is defective in patients with sepsis. Ascorbate or vitamin C, which is low in the plasma of patients with sepsis, is stored inside human neutrophils and is essential for their normal function.

Objective: This study aimed to determine if ascorbate treatment ex vivo improved neutrophil function in patients with sepsis.

Patients and Methods: Human blood neutrophils were isolated from 20 patients with sepsis and 20 healthy age-matched controls. Neutrophils were incubated with or without ascorbate $(1,5,10,20$ and $40 \mathrm{mM})$ for periods up to $2 \mathrm{~h}$. Chemotaxis was evaluated using a chemotactic chamber in response to the chemoattractant, fMLP. Phagocytosis (uptake of pHrodo red stained $S$. aureus) and apoptosis (annexin-V/propidium iodide staining) were measured by flow cytometry. Neutrophil extracellular trap (NET) formation was detected and quantified using DAPI, anti-myeloperoxidase and anti-neutrophil elastase immunofluorescence staining. Quantifluor detected the amount of dsDNA in NET supernatants, while quantitative PCR identified changes in expression of PADI4 gene.

Results: Chemotactic and phagocytic activities were decreased in patients with sepsis but increased after treatment with the high concentrations of ascorbate. Apoptosis was increased in the sepsis patients but not altered by ascorbate treatment. Spontaneous NET formation was observed in patients with sepsis. A quantity of $1 \mathrm{mM}$ ascorbate decreased spontaneous NETosis to that of normal, healthy neutrophils, while high concentrations of ascorbate $(>10 \mathrm{mM})$ further promoted NET formation.

Conclusion: Dysregulated neutrophil function was observed in patients with sepsis which could contribute to disease pathology and outcomes. Exposure to ascorbate could reverse some of these changes in function. These novel discoveries raise the possibility that ascorbate treatment could be used as an adjunctive therapy that could result in improved neutrophil function during sepsis.

Keywords: ascorbate, neutrophils, sepsis

\section{Plain Language Summary}

- Decreased neutrophil chemotaxis and phagocytosis were observed in patients with sepsis but this was improved by high concentrations of ascorbate.

- Patients with sepsis showed an increase in neutrophil apoptosis which did not change after ascorbate treatment.

- Patients with sepsis underwent high rates of spontaneous NETosis, that was decreased by $1 \mathrm{mM}$ of ascorbate treatment. 


\section{Introduction}

Neutrophils are the most abundant type of white blood cell in human circulation and their main function is to protect against invading pathogens by a variety of mechanisms such as chemotaxis, phagocytosis, apoptosis and neutrophil extracellular trap (NET) formation. ${ }^{1,2}$ There are several factors that maintain the effectiveness of neutrophil function, one of which is ascorbate or vitamin C. ${ }^{3,4}$ Ascorbate, a water-soluble vitamin, is essential for human homeostasis, metabolism and function of the immune system and is stored inside cytoplasm of neutrophils via vitamin $\mathrm{C}$ transporters. ${ }^{5-7}$ Although it has been reported that ascorbate promotes neutrophil chemotaxis and phagocytosis, ${ }^{8-10}$ its effects on NET formation and apoptosis are not well studied. ${ }^{4,11,12}$ In addition, it is unknown if ascorbate deficiencies or ascorbate supplementation can contribute to neutrophil function in inflammatory conditions or during infections such as sepsis, where neutrophil function may be compromised.

Although neutrophils are normally highly effective in eliminating invading pathogens, sometimes the invasion of pathogenic organisms overcomes this defence system, leading to systemic infection and sepsis. ${ }^{1,2,13,14}$ Sepsis is a life-threatening condition when the immune system, particularly neutrophils, vigorously battle against systemic infection. However, it has been reported that defective neutrophil function is observed in patients during sepsis, particularly elderly people with underlying illnesses and patients who are immune-compromised (eg, those with diabetes, heart diseases or malignancies). ${ }^{15}$

As ascorbate is an essential factor for neutrophil function, this study determined whether ascorbate treatment ex vivo enhanced neutrophil function in patients with sepsis. Neutrophil chemotaxis, phagocytosis, apoptosis and NET formation, including peptidyl arginine deiminase 4 or PADI4 gene expression (essential for the process of NET formation), were investigated in vitro. Here, we make the novel observation that short term (2h) ascorbate treatment can enhance several neutrophil functions that were impaired in sepsis patients. These novel observations raise the possibility that ascorbate treatment might be a useful adjunct therapy for sepsis patients.

\section{Patients and Methods}

The following reagents were used in this study: Polymorphprep (Axis-Shield, Norway); RPMI 1640 media with 25mM HEPES, L-Glutamine (Hyclone, USA); fetal bovine serum (Gibco, USA); sodium L-ascorbate (Sigma, USA); rabbit anti-neutrophil elastase and mouse antimyeloperoxidase and Alexa Fluor 488 conjugated goat antirabbit IgG and Alexa Fluor 647 conjugated anti-mouse IgG (Abcam, UK); 4',6-Diamidino-2-phenylindole dihydrochloride (Merck, USA); micrococcal nuclease from $S$. aureus (Sigma, USA); QuantiFluor one dsDNA (picogreen), (Promega, USA); Trizol ${ }^{\mathrm{TM}}$ Reagent (Ambion, USA); iScript $^{\mathrm{TM}}$ RT supermix; SsoAdvanced ${ }^{\mathrm{TM}}$ Universal SYBR Green Supermix (BIO-RAD, USA); PADI4 PCR primers (forward: 5'-CGAAGACCCCCAAGGACT-3', reverse: 5'AGGACAGTTTGCCCCGTG-3') and ITGB2 PCR primers (forward: 5'-GCTGTCCCCACAAAAAGTG-3', reverse: 5'CCGGAAGGTCACGTTGAA-3') and $\beta$-actin PCR primers (forward: 5'-TTCCTG GGCATGGAGTC-3', reverse: 5'CAGGTCTTTGCGGATGTC- $3^{\prime}$ ) (Integrated DNA Technology, Singapore); fMLP and Millipore Hanging Cell Culture plate inserts (MERCK, USA); Trypan blue (Sigma, USA); Annexin V-APC and propidium iodide (Biolegend, USA); pHrodo ${ }^{\mathrm{TM}}$ Red S. aureus Bioparticles Phagocytosis Kits (Invitrogen, USA).

\section{Patient and Healthy Controls}

Twenty patients diagnosed with sepsis using Sepsis-3 criteria, ${ }^{16-18}$ who attended the Emergency Department, Intensive Care Unit or Intermediate Intensive Care Unit, Inpatient Department, and 20 healthy aged-match controls were randomly recruited from the King Chulalongkorn Memorial Hospital, Thailand. Table 1 presents the demographics, underlying illnesses and identified organisms in hemoculture specimens in the patients. The levels of ascorbate (vitamin C) in plasma from patients with sepsis and healthy controls were measured using High Performance Liquid Chromatography (HPLC, Chromosystem, Germany) and shown in Table 1. Other detailed information of patients diagnosed with sepsis including blood chemistry, blood coagulation status, platelet count, source of infection and Sequential Sepsis-Related Organ Failure Assessment (SOFA) score is presented in Supplementary Table 1. This study was approved by Chulalongkorn University Human Research Ethic Committee (IRB 113/60) with validity date from 18 May 2018 until 17 May 2019. Written informed consent and/or assent forms were obtained from all donors.

\section{Isolation of Neutrophils}

The blood samples were collected within $72 \mathrm{~h}$ after the patients had been diagnosed with sepsis. Neutrophil isolation and culture method were described in our previous 
Table I Demographic Data

\begin{tabular}{|c|c|c|c|}
\hline Characteristics & Healthy $(\mathrm{N}=20)$ & Sepsis $(\mathbf{N}=20)$ & p-value \\
\hline \multicolumn{4}{|l|}{ Gender, n (\%) } \\
\hline Male & II (55) & II (55) & 1.0 \\
\hline Female & $9(45)$ & $9(45)$ & 1.0 \\
\hline Mean age, years $\pm S D$ & $61 \pm 14.1$ & $60 \pm 12.2$ & 1.0 \\
\hline $\begin{array}{l}\text { Time of diagnosis: days after } \\
\text { hospitalization; Median (IQ range) }\end{array}$ & $N / A$ & II (6-28) & $N / A$ \\
\hline WBC count $\left(\times 10^{3}\right.$ cells $\left./ \mu \mathrm{L} \pm \mathrm{SD}\right)$ & $6.7 \pm 2.3$ & $16.5 \pm 9.6$ & $<0.001$ \\
\hline Absolute neutrophil & $4.1 \pm 2.0$ & $14.3 \pm 9.4$ & $<0.001$ \\
\hline \multicolumn{4}{|l|}{ Underlying illnesses, n (\%) } \\
\hline Diabetes mellitus & $6(30)$ & $5(25)$ & $>0.99$ \\
\hline Hypertension & $6(30)$ & $7(35)$ & $>0.99$ \\
\hline Ischemic heart disease & $0(0)$ & $2(10)$ & 0.49 \\
\hline Dyslipidemia & $3(15)$ & $\mathrm{I}(5)$ & 0.61 \\
\hline Malignancy & $0(0)$ & $8(40)$ & 0.003 \\
\hline \multicolumn{4}{|l|}{ Organisms (identified in blood) } \\
\hline Staphylococcus spp. & $\mathrm{N} / \mathrm{A}$ & $\mathrm{I}(5)$ & $\mathrm{N} / \mathrm{A}$ \\
\hline Escherichia spp. & $N / A$ & $2(10)$ & $N / A$ \\
\hline Candida spp. & N/A & $2(10)$ & $N / A$ \\
\hline Unidentified organisms & $\mathrm{N} / \mathrm{A}$ & $15(75)$ & $\mathrm{N} / \mathrm{A}$ \\
\hline Plasma level of ascorbate or vitamin $\mathrm{C}(\mathrm{mg} / \mathrm{L} \pm \mathrm{SD})$ & $7.79 \pm 3.86$ & $1.03 \pm 2.07$ & $<0.01$ \\
\hline
\end{tabular}

Abbreviations: SD, standard deviation; WBC, white blood cell; N/A, not applicable.

studies. ${ }^{19,20}$ In brief, neutrophils were isolated from heparinised peripheral blood of patients with sepsis and healthy donors using Polymorphprep, according to the manufacturer's instructions. Red blood cell contamination was removed by hypotonic lysis buffer. Neutrophils were resuspended in RPMI 1640 media and the purity was assessed by staining with Wright stain and was $>95 \%$. Resuspended neutrophils were incubated at $37^{\circ} \mathrm{C}$ in a $5 \%$ $\mathrm{CO}_{2}$ incubator, with or without $10 \%(\mathrm{v} / \mathrm{v})$ fetal bovine serum, as indicated in the text.

\section{Neutrophil Chemotaxis Assay}

The chemotaxis assay was performed using 24-well tissue culture plates. Isolated neutrophils were treated with or without different concentrations of ascorbate for $2 \mathrm{~h}$ in a $5 \% \mathrm{CO}_{2}$ incubator. Chemoattractant (fMLP) was added into the wells, and the hanging inserts with a $3 \mu \mathrm{m}$ poresize filter were suspended in the culture media. Neutrophils at $10^{6}$ cells $/ \mathrm{mL}$ were added into the hanging inserts and incubated for $90 \mathrm{~min}$ at $37^{\circ} \mathrm{C}$ in a $5 \% \mathrm{CO}_{2}$ incubator. The hanging inserts were then removed and migrated neutrophils in each well were counted using the Countess $^{\mathrm{TM}}$ II automated cell counter (Thermo Fisher Scientific). Trypan blue staining was performed for detection of viable cells.

\section{Neutrophil Phagocytosis}

Phagocytic activity was measured using pHrodo $^{\mathrm{TM}}$ Red S. aureus Bioparticles Phagocytosis Kits. ${ }^{21}$ Isolated neutrophils were treated with or without different concentrations of ascorbate for $2 \mathrm{~h}$ at $37^{\circ} \mathrm{C}$ in a $5 \% \mathrm{CO}_{2}$ incubator. The cells were incubated with Bioparticles for $30 \mathrm{~min}$ in the incubator. The cells were washed and resuspended with PBS. Neutrophil phagocytosis was analysed on a flow cytometer (FACsAria II, BD Biosciences, USA) measuring 20,000 events per sample.

\section{Neutrophil Apoptosis}

After $2 \mathrm{~h}$ incubation in the presence or absence of different concentrations of ascorbate, neutrophils at $2.5 \times 10^{4}$ cells were stained with Annexin V-APC $(10 \mu \mathrm{L} / \mathrm{mL})$ for $15 \mathrm{~min}$ before they were stained with propidium iodide $(1 \mu \mathrm{g} / \mathrm{mL})$ 
as described previously. ${ }^{22}$ Stained cells were then analysed on a flow cytometer (FACsAria II, BD Biosciences, USA) analysing 20,000 events per sample.

\section{Neutrophil Extracellular Trap (NET) Assay}

Sterile round glass cover slips were placed into each well of a 24-well cell culture plate. Neutrophils $\left(5 \times 10^{5}\right.$ cells $)$ were added to each well and incubated for $1 \mathrm{~h}$ at $37^{\circ} \mathrm{C}$ in a $5 \% \mathrm{CO}_{2}$ incubator. Different concentrations of ascorbate (1, 5, 10, 20 and $40 \mathrm{mM}$ ) or $600 \mathrm{nM}$ phorbol myristate acetate (PMA) were added into the wells and incubated for $2 \mathrm{~h}$. The culture media was gently aspirated and the cover slips were washed with PBS. The cells and NETs were fixed with $1 \%$ formaldehyde. The glass cover slips were removed and incubated with $0.05 \%$ Tween in $1 \times$ PBS at room temperature for $1 \mathrm{~min}$ to permeabilize the cells. The cells were blocked for $30 \mathrm{~min}$ with 1xTBS with $2 \%$ bovine serum albumin.

NET formation was detected using immunofluorescence. Primary antibodies (rabbit anti-Neutrophil Elastase and mouse anti-Myeloperoxidase) were added (at 1:100 dilution) and incubated for 30min at room temperature. After washing in 1xTBS, secondary antibodies (anti-rabbit IgG and anti-mouse IgG) were added (at 1:400 dilution) and incubated for further $30 \mathrm{~min}$. The cover slips were washed and then stained with DAPI $(1 \mu \mathrm{g} / \mathrm{mL})$ before NET identification using a fluorescence microscope. ${ }^{23}$ The number of NETing cell was counted per 100 cells.

\section{Quantification of NET Formation (QuantiFlour ${ }^{\circledR} \mathrm{dsDNA}$ )}

Neutrophils were incubated exactly as described in 2.3 (in the absence and presence of ascorbate at the indicated concentrations) and incubated for $2 \mathrm{~h}$. After this incubation period, $0.1 \mathrm{M} \mathrm{CaCl}_{2}$ was added, followed by $500 \mathrm{mU}$ of micrococcal nuclease for $10 \mathrm{~min}$ to digest the NET structure and fragment the DNA. EDTA $(0.5 \mathrm{M})$ was added to inhibit the reaction and supernatants containing DNA were collected. Quantifluor (PicoGreen) was added to the supernatants, according to the manufacturer's instructions and incubated at room temperature for $5 \mathrm{~min}$ in the dark. The amount of DNA in the mixture was measured at $485 \mathrm{~nm}$ excitation (535 nm emission) on a PROMEGA Quantus ${ }^{\mathrm{TM}}$ Fluorometer. ${ }^{23}$

\section{PADI4 mRNA Expression}

Total RNA from isolated neutrophils was extracted using Trizol $^{\mathrm{TM}}$ Reagent followed by a DNase digestion step, according to the manufacturer's instruction. ${ }^{19}$ The extracted RNA was converted to cDNA using iScript ${ }^{\mathrm{TM}}$ RT supermix. PADI4 gene expression was detected by quantitative PCR using SsoAdvanced ${ }^{\mathrm{TM}}$ Universal SYBR Green Supermix (BIO-RAD), ${ }^{24}$ using a 7500 ABI RealTime PCR System.

\section{Statistical Analysis}

Statistical analyses were performed by using GraphPad version 7, Student's $t$-test and one-way ANOVA test. Data are expressed as mean $\pm \mathrm{SEM}$, and differences with a p-value of $<0.05$ were considered statistically significant.

\section{Results}

\section{Neutrophil Chemotaxis, Phagocytosis and Apoptosis in Patients with Sepsis}

Neutrophils isolated from patients with sepsis showed significantly decreased cell migration $(13.6 \pm 8.5 \%$, $\mathrm{n}=10, \mathrm{p}<0.01)$ in a response towards the chemoattractant fMLP $(100 \mathrm{nM})$, compared to healthy control neutrophils $(31.3 \pm 10.7 \%, \mathrm{n}=10)$ (Figure $1 \mathrm{~A}$ and $\mathrm{B}$ ). Phagocytic activity of neutrophils isolated from patients with sepsis was significantly decreased (15.4 $\pm 2.6 \%, \mathrm{n}=5, \mathrm{p}<0.01)$, compared with healthy controls $(34.4 \pm 15.3 \%, \mathrm{n}=5)$ (Figure $1 \mathrm{C}$ and D). Increased neutrophil apoptosis was observed in patients with sepsis $(15.6 \pm 2.3 \%, \mathrm{n}=5, \mathrm{p}<0.005)$ compared to healthy controls $(7.3 \pm 1.5 \%, \mathrm{n}=5)$ when measured $2 \mathrm{~h}$ post-isolation (Figure 1E and $\mathrm{F}$ ).

\section{Spontaneous NET Formation in Patients with Sepsis}

Neutrophils isolated from patients with sepsis showed significantly increased capacity for spontaneous NET formation after $2 \mathrm{~h}$ post-isolation $(21.7 \pm 18.7$ cells $/ 100$ cells undergoing NETosis, $\mathrm{n}=20, \mathrm{p}<0.001$ ) compared to healthy control neutrophils which showed only barely detectable levels of NETosis $(1.2 \pm 0.9$ cells $/ 100$ cells, $n=20)$ (Figure $2 \mathrm{~A}$ and $\mathrm{B})$. Immuno-fluorescence staining confirmed the presence of both elastase and myeloperoxidase on these DNA structures, confirming the formation of genuine NETs (Figure 2A). Induced NET formation by PMA as a positive control is shown in Supplementary Figure 1.

NETs contain double-stranded DNA (dsDNA), ${ }^{25,26}$ and so we quantified the amount of released dsDNA from sepsis patients and healthy donors. The results showed that the levels of dsDNA were significantly increased in 
A
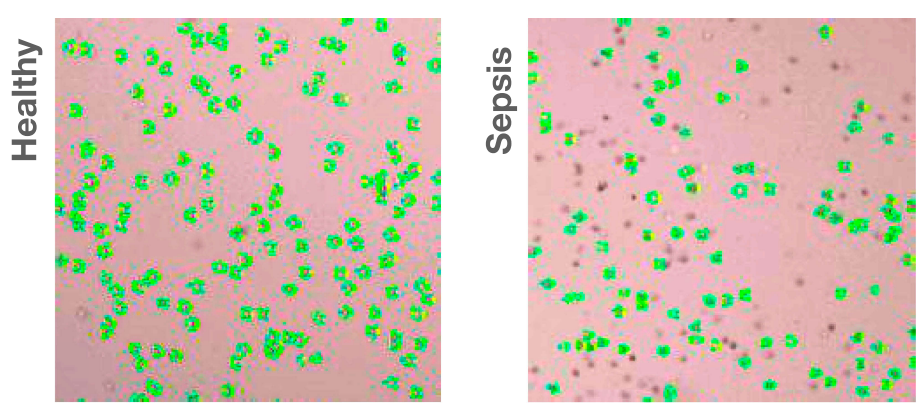

C
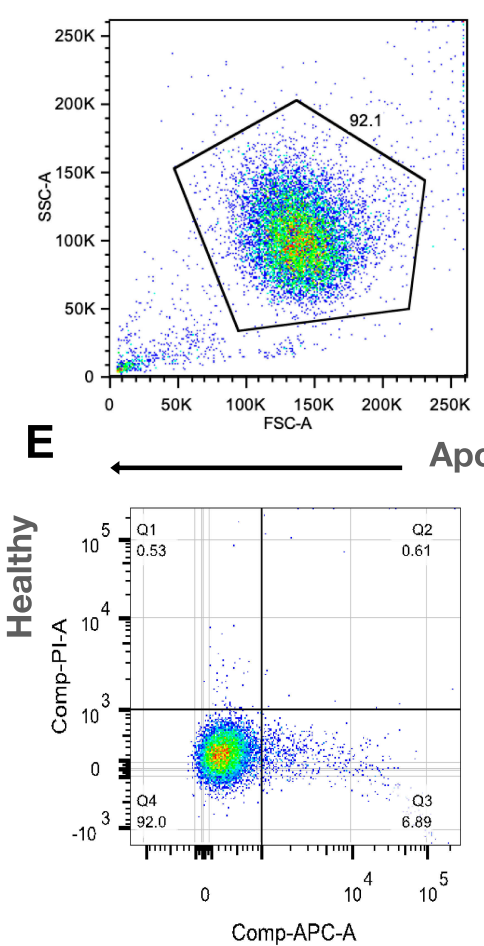

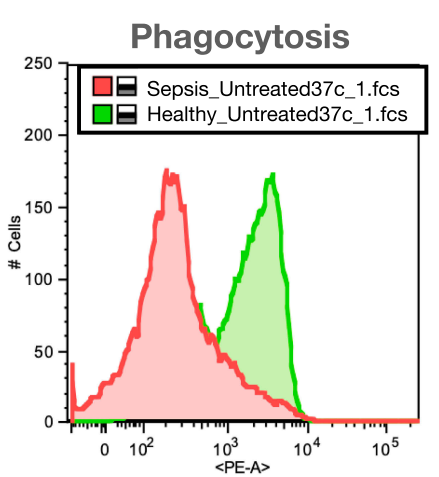

Apoptosis

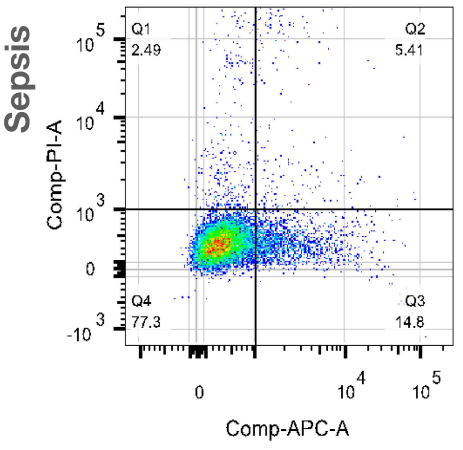

B

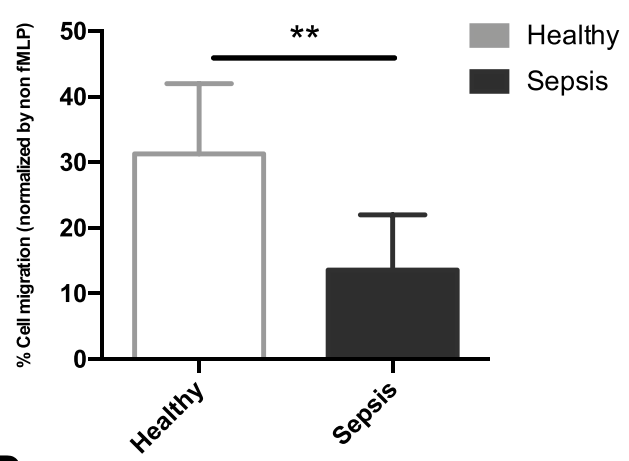

D
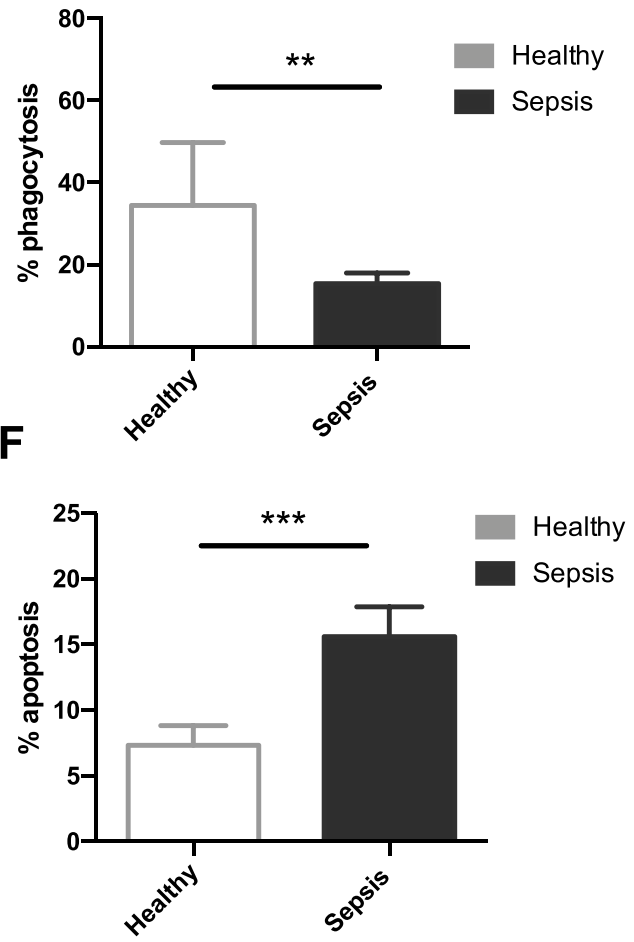

Figure I Representative images of neutrophils from cell migration stained with trypan blue and counted by automated cell counter (A), and the percentages of neutrophil chemotaxis of healthy donors $(n=10)$ and patients with sepsis $(n=10)(B)$. Representative flow cytometry results $(\mathbf{C})$, and the percentages of phagocytic activity of healthy donors $(n=5)$ and patients with sepsis $(n=5)(D)$. Representative flow cytometry results $(E)$, and the percentages of neutrophil apoptosis of healthy donors ( $=5)$ and patients with sepsis $(n=5)(\mathbf{F})$.

Notes: ${ }^{* *} \mathrm{p}<0.01$, *** $\mathrm{p}<0.005$.

patients with sepsis $(0.94 \pm 0.24 \mathrm{ng} / \mathrm{mL}, \mathrm{n}=10, \mathrm{p}<0.01)$ compared to healthy control neutrophils $(0.63 \pm 0.14 \mathrm{ng} /$ $\mathrm{mL}, \mathrm{n}=10$ ) (Figure 2C). To confirm these observations, we measured mRNA expression of PADI4 gene as PAD4 enzyme is required for the process of NET formation. ${ }^{12,27}$ The results showed that PADI4 mRNA expression was significantly increased in patients with sepsis $(7.6 \pm 3.3, \mathrm{n}=10, \mathrm{p}<0.05)$, compared to healthy control neutrophils $(3.9 \pm 2.2, \mathrm{n}=10)$ (Figure 2D).
Neutrophil Chemotaxis, Phagocytosis and Apoptosis After Ascorbate Treatment in Patients with Sepsis

After $2 \mathrm{~h}$ treatment with different concentrations of ascorbate, the percentages of cell migration when neutrophils were treated with high concentrations $(10,20$ and $40 \mathrm{mM})$ of ascorbate were significantly increased in both sepsis patients $(51.9 \pm 10.8 \%, 67.7 \pm 15.6 \%$ and $59.5 \pm 9.9 \%$, 
A
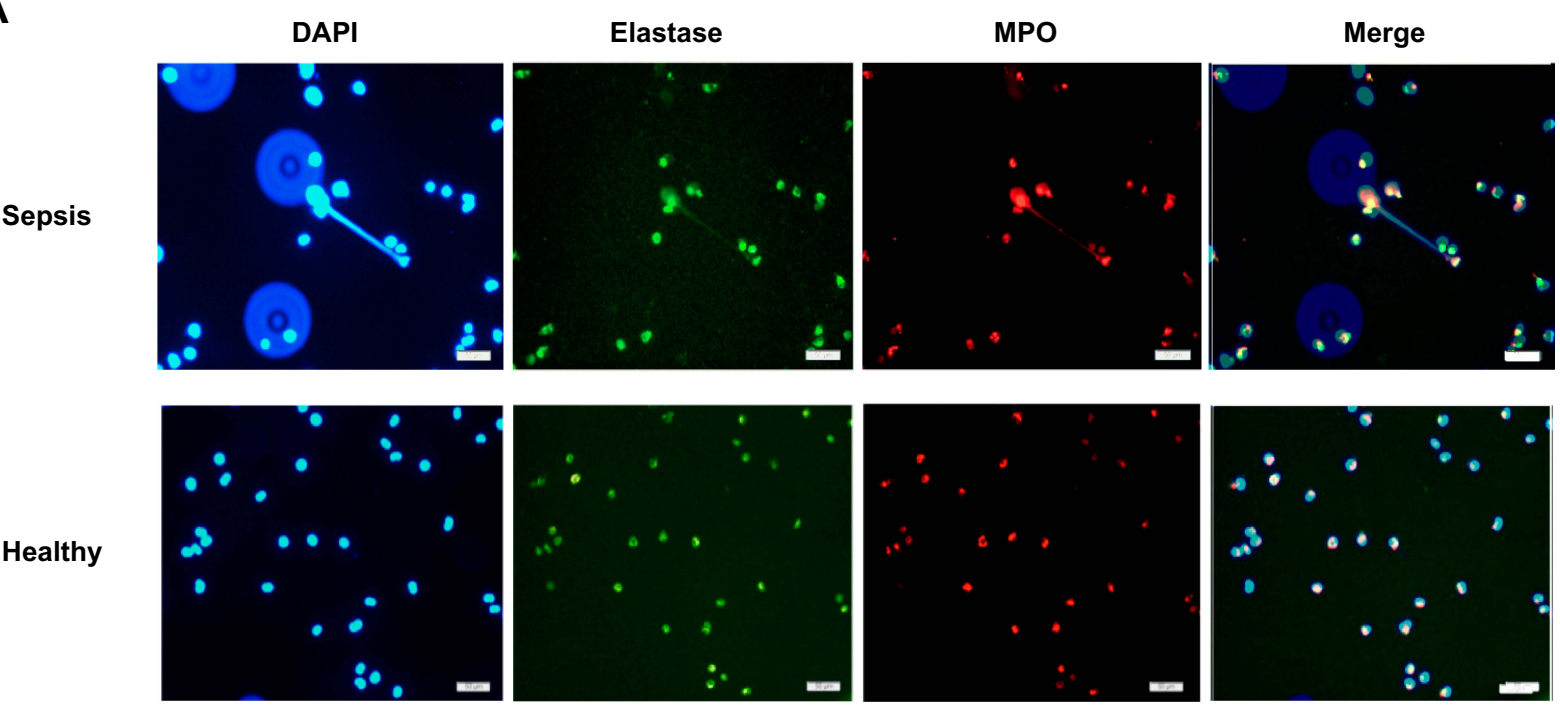

B

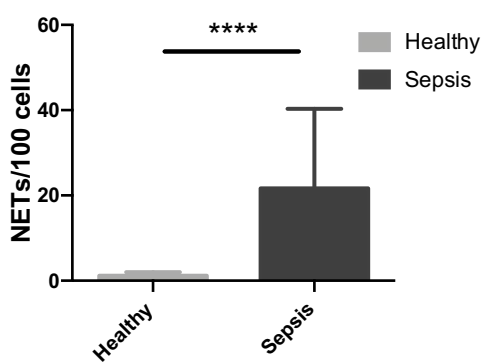

C

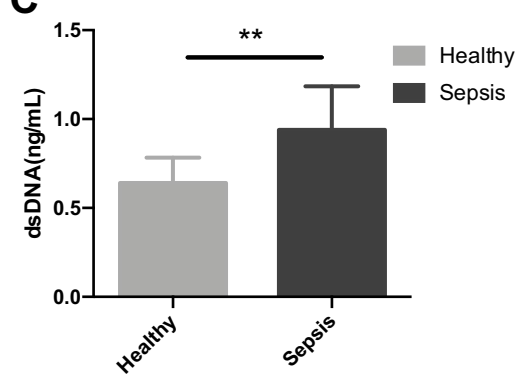

D

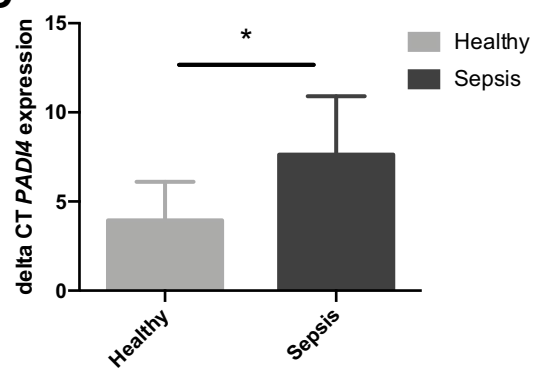

Figure 2 Representative fluorescence images (400X) of isolated neutrophils from healthy controls $(n=20)$ and patients with sepsis $(n=20)$, stained with DAPI (blue), elastase (green) and myeloperoxidase (red), and merged images for NET identification after $2 \mathrm{~h}$ post-isolation ex vivo (A). Spontaneous NET formation after $2 \mathrm{~h}$ post-isolation (B), the levels of dsDNA (C) and PADI4 mRNA expression (D) from healthy controls and patients with sepsis ( $n=10$ for both groups).

Notes: ${ }^{*} p<0.05,{ }^{*} p<0.01, * * * * p<0.001$.
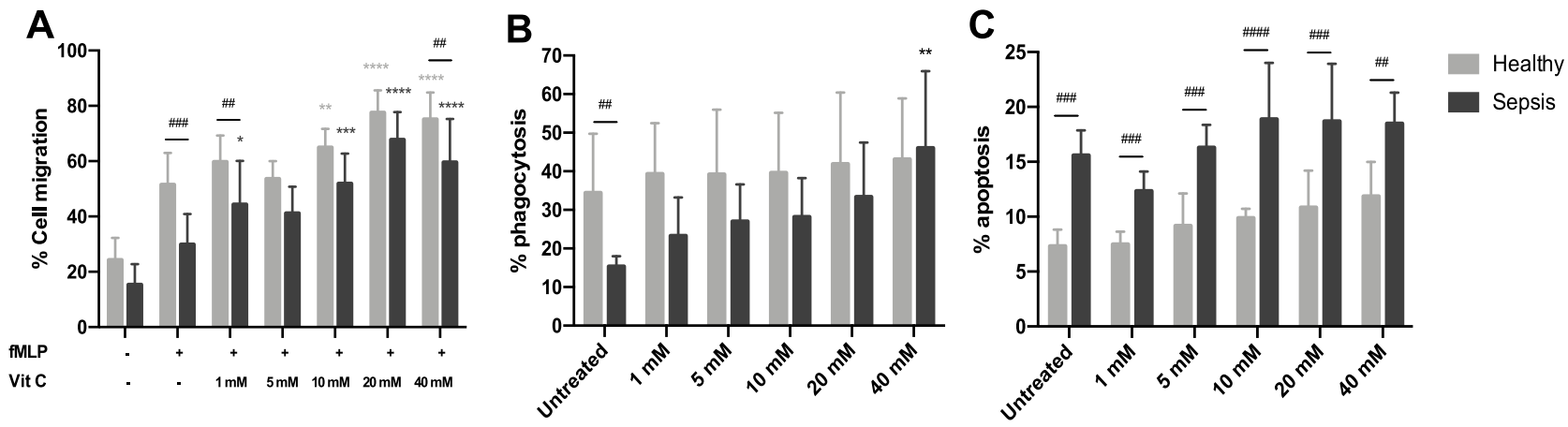

Figure 3 The percentages of neutrophil chemotaxis or cell migration $(\mathbf{A})$, phagocytosis $(\mathbf{B})$ and apoptosis $(\mathbf{C})$ from healthy donors and patients with sepsis $(\mathrm{n}=5$ for both groups) and the effect of ascorbate (I, 5, 10, 20 and $40 \mathrm{mM})$ on neutrophils after $2 \mathrm{~h}$ treatment.

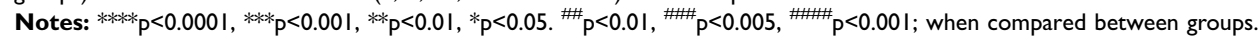

respectively) and healthy neutrophils $(65.0 \pm 6.7 \%, 77.5 \pm$ $8.1 \%$, and $75.1 \pm 9.7 \%$, respectively), compared to sepsis and healthy neutrophils $(29.9 \pm 11.0 \%$ and $51.5 \pm 11.4 \%$, respectively, $\mathrm{n}=5$ for both groups, $\mathrm{p}<0.001$ ) (Figure $3 \mathrm{~A}$ ).
Of note, the increased rates of neutrophil chemotaxis in sepsis patients and healthy controls were comparable at each concentration of ascorbate ( $\mathrm{p}>0.05)$, except only at 1 and $40 \mathrm{mM}(\mathrm{p}<0.01)$. 
Ascorbate treatment increased neutrophil phagocytosis in sepsis patients but this increase was significantly enhanced at a concentration of $40 \mathrm{mM}(46.1 \pm 19.8 \%$, $\mathrm{n}=5, \mathrm{p}<0.01$ ) when compared with untreated neutrophils $(15.4 \pm 2.6 \%, n=5)$ (Figure 3B). However, we did not observe an increase in phagocytosis from healthy control neutrophils after ascorbate treatment $(n=5, p>0.05)$. In addition, no significant differences in neutrophil phagocytosis were found between sepsis patients and healthy controls at each concentration of ascorbate treatment $(\mathrm{p}>0.05)$.

The percentages of neutrophil apoptosis in sepsis patients and healthy controls were unaffected by $2 \mathrm{~h}$ treatment with ascorbate at all concentrations tested $(\mathrm{n}=5$ for both groups, $\mathrm{p}>0.05$ ) (Figure $3 \mathrm{C}$ ). These percentages of neutrophil apoptosis were still significantly increased in patients with sepsis compared with healthy controls at every concentrations of ascorbate treatment $(\mathrm{p}<0.01)$.

\section{NET Formation After Ascorbate \\ Treatment in Patients with Sepsis}

After $2 \mathrm{~h}$ treatment with different concentrations of ascorbate, the level of NET formation from neutrophils incubated with 1 $\mathrm{mM}$ ascorbate was significantly lower $(4.4 \pm 3.1$ cells $/ 100$ cells, $\mathrm{n}=10, \mathrm{p}<0.05$ ) in patients with sepsis, compared to untreated sepsis neutrophils $(21.7 \pm 18.7$ cells $/ 100$ cells, $\mathrm{n}=10$ ) (Figure 4A and B). However, the levels of dsDNA and PADI4 mRNA expression from sepsis neutrophils treated with $1 \mathrm{mM}$ were decreased but these decreases did not reach statistical significances ( $p>0.05$ ) (Figure $4 \mathrm{C}$ and $\mathrm{D}$ ). Furthermore, the levels of NET formation, dsDNA and

A
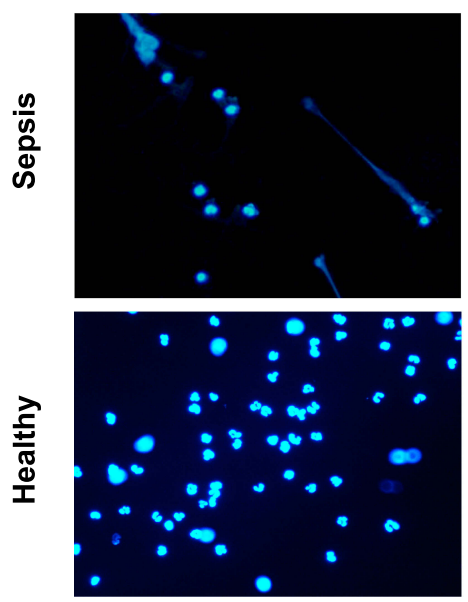

B

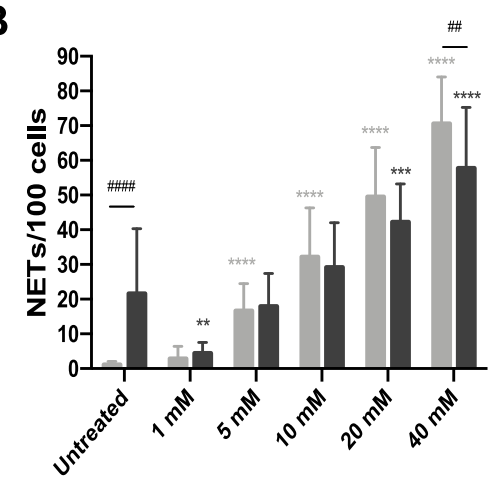

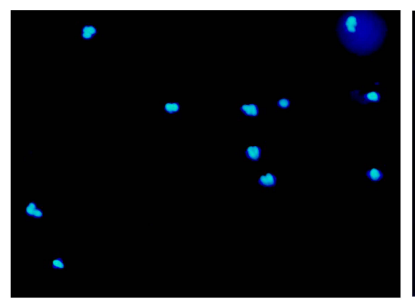
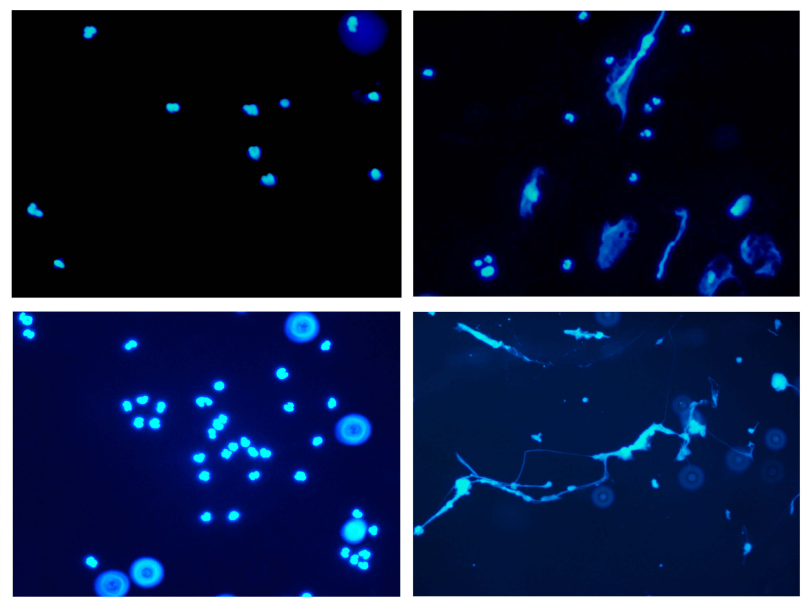

C

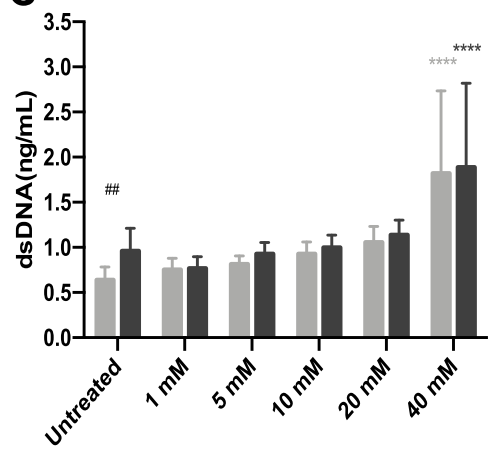

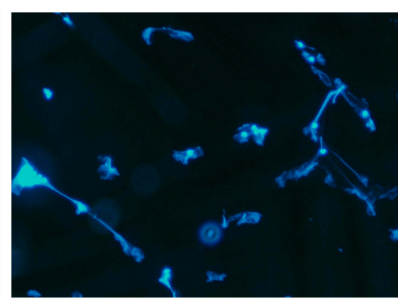

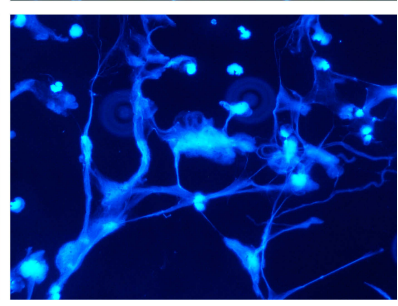

D

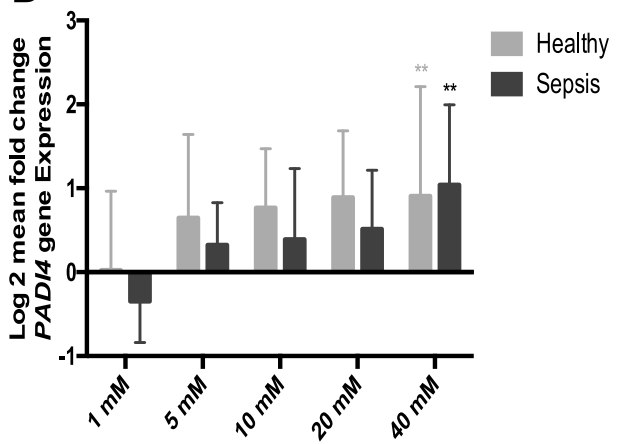

Figure 4 Effect of ascorbate on NET formation. Representative fluorescence images (400X) of isolated neutrophils from patients with sepsis and healthy controls treated with $\mathrm{I}, 10$ and $40 \mathrm{mM}$ of ascorbate for $2 \mathrm{~h}$ and stained with DAPI staining for NET identification (A). NETs counted per 100 neutrophils ( $\mathrm{n}=20$ for both groups) (B), the levels of dsDNA (C) and PADI4 mRNA expression (D) from patients with sepsis and healthy controls ( $\mathrm{n}=10$ for both groups) treated with different concentrations of ascorbate $(\mathrm{I}, 5,10,20$ and $40 \mathrm{mM})$ for $2 \mathrm{~h}$.

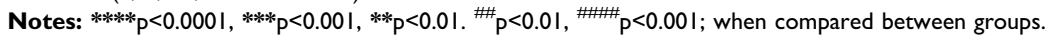


PADI4 mRNA expression between sepsis patients and healthy controls were comparable when their neutrophils were treated with $1 \mathrm{mM}$ of ascorbate (Figure 4B, 4C and D) ( $>>0.05)$.

In contrast, higher levels of NET formation were observed in sepsis neutrophils after ascorbate treatment $(\geq 5$ $\mathrm{mM}$ ) particularly at the concentrations of 20 and $40 \mathrm{mM}$ $(42.3 \pm 10.9$ and $57.8 \pm 17.4$ NETs/100 cells, respectively, $\mathrm{p}<0.001)$, compared to untreated sepsis neutrophils $(21.7 \pm$ 18.7 NETs/100 cells, $n=20$ for both groups). This observation was also observed in healthy neutrophils after ascorbate treatment ( $\geq 5 \mathrm{mM})$, compared to untreated healthy neutrophils $(\mathrm{p}<0.001)$ (Figure 4B). The levels of NET formation expression between sepsis patients and healthy controls were comparable at every concentrations of ascorbate treatment ( $\mathrm{p}>0.05)$, except at $40 \mathrm{mM}(\mathrm{p}<0.01)$.

The levels of dsDNA in supernatants were significantly increased in sepsis and healthy control neutrophils at 40 $\mathrm{mM}$ of ascorbate treatment $(1.9 \pm 0.95$ and $1.8 \pm 0.9 \mathrm{ng} /$ $\mathrm{mL}$, respectively, $\mathrm{n}=10$ ), compared to untreated neutrophils $(0.94 \pm 0.24 \mathrm{ng} / \mathrm{mL}$ and $0.63 \pm 0.14 \mathrm{ng} / \mathrm{mL}$, respectively, $\mathrm{n}=10, \mathrm{p}<0.01$ ) (Figure $4 \mathrm{C}$ ). In addition, increased levels of PADI4 mRNA expression were also detected in both groups after their neutrophils were treated with 40 $\mathrm{mM}$ of ascorbate $(1.04 \pm 0.95$ and $0.72 \pm 0.30$, respectively, $n=10)$, compared to untreated neutrophils $(\mathrm{p}<0.01)$ (Figure 4D). No significance differences in the levels of dsDNA and PADI4 mRNA expression between sepsis patients and healthy controls were found at every concentration of ascorbate treatment $(\mathrm{p}>0.05)$ (Figure $4 \mathrm{C}$ and D).

\section{Discussion}

Sepsis is a complex clinical syndrome that develops once a local infection becomes uncontrollable and the causative pathogens invade into the bloodstream, leading to systemic inflammation and multi-organ dysfunction. ${ }^{13-15}$ Early clinical diagnosis and prompt treatments are crucial to improve outcomes of patients with sepsis, but the overall mortality rate is tremendously high, particularly in immune-compromised hosts and elderly patients with multiple underlying illnesses. ${ }^{28-30}$

In our study, patients were diagnosed with sepsis using the clinical criteria of Sepsis-3. Each patient had both an identified source of infection and an acute change in total SOFA score $\geq 2$ points. However, some patients showed unidentified organisms in their hemocultures and this observation was probably because of slow-growing or intracellular organisms, or antibiotic treatment started before blood sampling. ${ }^{31-33}$
Neutrophils are the major innate immune cell that play a role in the pathogenesis of sepsis, and previous studies have demonstrated that neutrophil function is dysregulated in these patients. ${ }^{15}$ Moreover, ascorbate, an important factor that maintains neutrophil function, rapidly declines in the plasma of neutrophils during sepsis. ${ }^{7,34-37}$ In our study, we also found that the plasma level of ascorbate in patients with sepsis was significantly lower than the levels in healthy controls $(<0.01)$ as shown in Table 1 . Therefore, it may be hypothesised that ascorbate supplementation could restore impaired neutrophil function in these patients.

Our study, therefore, set out to first determine the function of neutrophils isolated from sepsis patients. Neutrophil chemotaxis is the crucial step in the inflammatory response to invading pathogens and phagocytosis is the main killing mechanism of neutrophils. In our study, chemotaxis and phagocytosis were decreased in patients with sepsis which is consistent with previous studies. ${ }^{38-41}$ Furthermore, delayed neutrophil apoptosis is the final step in the cycle of neutrophil activation and the resolution of inflammation, ${ }^{42}$ and neutrophils become apoptotic prior to removal by phagocytic cells once they have finished pathogen clearance. However, the rate of neutrophil apoptosis in vivo in patients with sepsis is still unknown. ${ }^{43,44}$ Our study demonstrated increased spontaneous neutrophil apoptosis $2 \mathrm{~h}$ post-isolation of neutrophils from patients with sepsis. Our findings support the idea that neutrophil function is abnormal in patients with sepsis and reduced neutrophil migration may be partially due to increased apoptosis.

Our next experiments measured the capacity of neutrophils from sepsis patients to undergo spontaneous NETosis. This function is an important killing mechanism of neutrophils against invading pathogens, particularly in the event that extracellular pathogens cannot be appropriately phagocytosed. However, inappropriate NET formation may also contribute to inflammation and autoimmunity, for example, by exposure of auto-antigens (eg, granule contents) or neo-antigens that are generated via post-translational modification of nuclear proteins (eg, citrullinated or acetylated histones). ${ }^{45}$ After neutrophil activation, NETs are formed and released extracellularly in order to trap and inhibit systemic spreading of the organisms using the web-like DNA structure. Subsequently, the proteolytic enzymes (eg, neutrophil elastase) and myeloperoxidase kill the trapped microbes. ${ }^{15}$ The role of NETosis in sepsis is complex in that NET 
formation may initially help prevent the spread and dissemination of bacteria from a localized site of infection, thereby limiting systemic infection. ${ }^{46}$ Nevertheless, excessive NET formation in the later stages of sepsis may play a role in the development of thrombosis and organ failure. $^{47}$

In our study, we showed that neutrophils isolated from patients with sepsis developed spontaneous NET formation over a $2 \mathrm{~h}$ incubation ex vivo. This observation is consistent with a previous study, suggesting that neutrophils were already stimulated by the pathogens infecting the patients during sepsis. ${ }^{48}$ This finding was confirmed by measurements of an increase in dsDNA levels and upregulation of PADI4 mRNA expression, a key enzyme involved in the regulation of NETosis, ${ }^{49}$ in neutrophils isolated from patients with sepsis. However, we observed a very high range of spontaneous NETosis in these sepsis patients, ranging from $2 \%$ to $3 \%$ of the neutrophils to over $80 \%$ of the cells undergoing NETosis. These reasons for this very large variation in NETosis are unknown, and we could not find any association between the extent of NETosis and any clinical parameters, tested such as severity of disease, type of bacterial infection, and duration of sepsis or treatment.

Therefore, the effect of ascorbate on neutrophil functions were investigated in our study. We found that high concentrations of ascorbate treatment (particularly at 40 $\mathrm{mM}$ ) increased both neutrophil chemotactic and phagocytic activities in these patients. Similar findings have been reported in both sepsis patients and mouse models, which showed increased chemotaxis and phagocytosis after ascorbate supplementation. ${ }^{10,50,51}$ Moreover, neutrophil chemotaxis and phagocytosis in healthy controls were increased by ascorbate treatment. Thus, the effects of ascorbate on neutrophil function are not restricted to cells isolated from sepsis patients, but rather are more generalized effects on some neutrophil functions. Interestingly, we noticed that these neutrophil functions were comparable between sepsis patients and healthy controls after their neutrophils were treated with ascorbate $(\geq 5$ $\mathrm{mM}$ ), suggesting that neutrophil dysfunction in patients with sepsis were restored and returned to nearly normal function by ascorbate treatment. The potential mechanism of ascorbate on the enhancement of neutrophil chemotaxis and phagocytosis was reported in previous studies which indicated that intracellular microtubule assembly of neutrophil was stabilized by ascorbate treatment, leading to improvement of neutrophil motility and function. ${ }^{50,52}$
However, ascorbate treatment did not significantly change the rate of neutrophil apoptosis in our patients and healthy controls. Therefore, this finding suggests that neutrophil apoptosis is still required as the last step of eliminating pathogens in patients with sepsis, and ascorbate is not involved in the apoptotic process of neutrophils. In contrast, a previous study showed that intravenous ascorbate supplementation in patients with sepsis after abdominal surgery temporarily decreased the levels of apoptotic proteins in peripheral blood neutrophils. ${ }^{53}$ However, the rate of neutrophil apoptosis was not determined in their study.

Having shown that spontaneous NETosis was observed in sepsis patients, we then determined whether ascorbate could alter these levels of NETosis, as serum and plasma levels of ascorbate were shown to be decreased during sepsis in previous studies and our own patients. ${ }^{36} \mathrm{We}$ found that low level of ascorbate $(1 \mathrm{mM})$ could significantly decrease levels of spontaneous NETosis of neutrophils from sepsis patients, which was confirmed by decreased levels of PADI4 mRNA expression in these patients (Figure 4D). Moreover, we noticed that the levels of NETosis from both sepsis patients and healthy controls were less detectable ( $<5$ NETs counted in Figure 4B) and comparable (dsDNA levels in Figure 4C) after their neutrophils were treated with or without $1 \mathrm{mM}$ of ascorbate. Our findings suggest that $1 \mathrm{mM}$ of ascorbate treatment significantly reduces spontaneous NET formation in patients with sepsis and their rates of NETosis return to normal as seen in healthy controls. The potential mechanism of ascorbate on decreased NET formation may be due to the modulation of redox-related cell signaling pathways by ascorbate, which stabilizes and protects the cell membrane from oxidative stress during sepsis leading to a decrease in NET release. ${ }^{50}$

Higher concentrations of ascorbate $(>10 \mathrm{mM})$ further increased NETosis significantly in both patients with sepsis and healthy controls, above the already high levels observed in the absence of this compound. However, previous studies showed a significant decrease in NETs inside the lungs of ascorbate-deficient mice after ascorbate supplementation, ${ }^{54,55}$ which is contrast to our study, and probably explained by different tissue sources of neutrophils studied (lungs VS peripheral blood in our study and species variations) and different concentrations of ascorbate used in both studies.

The increased levels of NETosis after higher concentrations of ascorbate treatment were confirmed by 
measurements of an increase in dsDNA levels in culture supernatants and increased levels of PADI4 mRNA expression of neutrophils treated with ascorbate. We believe that these higher concentrations of ascorbate further induce an oxidative burst and activate the PAD4 enzyme leading to an increase in NET formation. ${ }^{56}$ Nevertheless, the levels of dsDNA at certain concentrations of ascorbate were not significantly different as seen under light microscopy, probably due to some limitations of the PicoGreen assay, ${ }^{57}$ and delayed upregulation of mRNA transcription and protein translation which probably need more future studies.

As integrin activation is associated with all neutrophil phenotypes observed in our study, we further investigated the activation status of integrins on neutrophils from patients with sepsis and healthy controls as shown in Supplementary Figure 2. As expected, an increase in expression of ITGB2 ( $\beta_{2}$-integrin) was observed in the patient group, probably because their neutrophils were stimulated by pathogens and cytokines during sepsis. ${ }^{58}$ However, the ITGB2 expression was unchanged after ascorbate treatment in both groups.

One limitation of this study was that the numbers of isolated neutrophils from patients or healthy controls were varied and sometimes they were not enough to perform every designed experiments. However, these numbers of patients were sufficient for statistical analyses.

In conclusion, our study demonstrated neutrophil dysfunction in patients with sepsis and ascorbate could improve the defective chemotaxis and phagocytosis observed in neutrophils from these patients. Interestingly, high levels of spontaneous NETosis from sepsis patients could be returned to normal by low concentrations of ascorbate (1 $\mathrm{mM})$. However, further studies are probably needed to investigate the mechanisms how NETosis, chemotaxis and phagocytosis, but not apoptosis, were enhanced by high concentrations of ascorbate, which have never been explored. ${ }^{50}$ This study suggests that ascorbate could potentially be used as an adjunctive treatment for patients during sepsis. However, as its effects are highly dose-dependent, such treatments should carefully examine the effective doses that are clinically beneficial and whether NETosis should be prevented or promoted in such patients.

\section{Acknowledgments}

We would like to thank all patients and healthy controls for their contributions to this study and acknowledge colleagues in the Institute of Integrative Biology, University of Liverpool, Liverpool, UK. Special thanks go to
Rachadapisek Sompod Endowment Foundation (RA60/ 034) and the Faculty of Medicine, Chulalongkorn University, Bangkok, Thailand for financial support.

\section{Author Contributions}

All authors made substantial contributions to conception and design, acquisition of data, or analysis and interpretation of data; took part in drafting the article or revising it critically for important intellectual content; gave final approval of the version to be published; and agree to be accountable for all aspects of the work.

\section{Disclosure}

The authors report no conflicts of interest in this work.

\section{References}

1. Kolaczkowska E, Kubes P. Neutrophil recruitment and function in health and inflammation. Nat Rev Immunol. 2013;13(3):159-175. doi:10.1038/nri3399

2. Papayannopoulos V. Neutrophil extracellular traps in immunity and disease. Nat Rev Immunol. 2018;18(2):134-147. doi:10.1038/ nri.2017.105

3. Vissers MC, Hampton MB. The role of oxidants and vitamin $\mathrm{C}$ on neutrophil apoptosis and clearance. Biochem Soc Trans. 2004;32 (Pt3):499-501. doi:10.1042/bst0320499

4. Chatterjee M, Saluja R, Kumar V, et al. Ascorbate sustains neutrophil NOS expression, catalysis, and oxidative burst. Free Radic Biol Med. 2008;45(8):1084-1093. doi:10.1016/j.freeradbiomed.2008.06.028

5. Chambial S, Dwivedi S, Shukla KK, John PJ, Sharma P. Vitamin C in disease prevention and cure: an overview. Indian J Clin Biochem. 2013;28(4):314-328. doi:10.1007/s12291-013-0375-3

6. Figueroa-Mendez R, Rivas-Arancibia S. Vitamin C in health and disease: its role in the metabolism of cells and redox state in the brain. Front Physiol. 2015;6:397. doi:10.3389/fphys.2015.00397

7. Anderson R, Oosthuizen R, Maritz R, Theron A, Van Rensburg AJ. The effects of increasing weekly doses of ascorbate on certain cellular and humoral immune functions in normal volunteers. Am J Clin Nutr. 1980;33(1):71-76. doi:10.1093/ajcn/33.1.71

8. Wang Y, Russo TA, Kwon O, Chanock S, Rumsey SC, Levine M. Ascorbate recycling in human neutrophils: induction by bacteria. Proc Natl Acad Sci U S A. 1997;94(25):13816-13819. doi:10.1073/ pnas.94.25.13816

9. Erickson KL, Medina EA, Hubbard NE. Micronutrients and innate immunity. J Infect Dis. 2000;182(Suppl 1):S5-10. doi:10.1086/ 315922

10. de la Fuente M, Ferrandez MD, Burgos MS, Soler A, Prieto A, Miquel J. Immune function in aged women is improved by ingestion of vitamins C and E. Can J Physiol Pharmacol. 1998;76(4):373-380. doi:10.1139/y98-038

11. Bozonet SM, Carr AC, Pullar JM, Vissers MC. Enhanced human neutrophil vitamin $\mathrm{C}$ status, chemotaxis and oxidant generation following dietary supplementation with vitamin C-rich SunGold kiwifruit. Nutrients. 2015;7(4):2574-2588. doi:10.3390/nu7042574

12. Mohammed BM, Fisher BJ, Kraskauskas D, et al. Vitamin C: a novel regulator of neutrophil extracellular trap formation. Nutrients. 2013;5 (8):3131-3151. doi:10.3390/nu5083131

13. Vincent JL, Opal SM, Marshall JC, Tracey KJ. Sepsis definitions: time for change. Lancet. 2013;381(9868):774-775. doi:10.1016/ S0140-6736(12)61815-7 
14. Hotchkiss RS, Karl IE. The pathophysiology and treatment of sepsis N Engl J Med. 2003;348(2):138-150. doi:10.1056/NEJMra021333

15. Shen XF, Cao K, Jiang JP, Guan WX, Du JF. Neutrophil dysregulation during sepsis: an overview and update. J Cell Mol Med. 2017;21 (9):1687-1697. doi:10.1111/jcmm.13112

16. Singer M, Deutschman CS, Seymour CW, et al. The third international consensus definitions for sepsis and septic shock (Sepsis-3). JAMA. 2016;315(8):801-810. doi:10.1001/jama.2016.0287

17. Kaukonen KM, Bailey M, Bellomo R. Systemic inflammatory response syndrome criteria for severe sepsis. $N$ Engl $J$ Med. 2015;373(9):881.

18. Seymour CW, Liu VX, Iwashyna TJ, et al. Assessment of clinical criteria for sepsis for the third international consensus definitions for sepsis and septic shock (Sepsis-3). JAMA. 2016;315(8):762-774. doi:10.1001/jama.2016.0288

19. Chiewchengchol D, Wright HL, Thomas HB, et al. Differential changes in gene expression in human neutrophils following TNF-alpha stimulation: up-regulation of anti-apoptotic proteins and down-regulation of proteins involved in death receptor signaling. Immun Inflamm Dis. 2016;4(1):35-44. doi:10.1002/iid3.90

20. Chiewchengchol D, Midgley A, Sodsai P, et al. The protective effect of GM-CSF on serum-induced neutrophil apoptosis in juvenile systemic lupus erythematosus patients. Clin Rheumatol. 2015;34 (1):85-91. doi:10.1007/s10067-014-2800-2

21. Ballantine L, Midgley A, Harris D, Richards E, Burgess S, Beresford MW. Increased soluble phagocytic receptors sMer, sTyro3 and sAxl and reduced phagocytosis in juvenile-onset systemic lupus erythematosus. Pediatr Rheumatol Online J. 2015;13:10. doi:10.1186/s12969-015-0007-y

22. Wlodkowic D, Skommer J, Darzynkiewicz Z. Flow cytometry-based apoptosis detection. Methods Mol Biol. 2009;559:19-32.

23. Wright HL, Makki FA, Moots RJ, Edwards SW. Low-density granulocytes: functionally distinct, immature neutrophils in rheumatoid arthritis with altered properties and defective TNF signalling. J Leukoc Biol. 2017;101(2):599-611. doi:10.1189/jlb.5A0116-022R

24. Dong S, Zhang Z, Takahara H. Estrogen-enhanced peptidylarginine deiminase type IV gene (PADI4) expression in MCF-7 cells is mediated by estrogen receptor-alpha-promoted transfactors activator protein-1, nuclear factor-Y, and Sp1. Mol Endocrinol. 2007;21 (7):1617-1629. doi:10.1210/me.2006-0550

25. Margraf S, Logters T, Reipen J, Altrichter J, Scholz M, Windolf J. Neutrophil-derived circulating free DNA (cf-DNA/NETs): a potential prognostic marker for posttraumatic development of inflammatory second hit and sepsis. Shock. 2008;30(4):352-358. doi:10.1097/SHK.0b013e31816a6bb1

26. Lee KH, Cavanaugh $\mathrm{L}$, Leung $\mathrm{H}$, et al. Quantification of NETs-associated markers by flow cytometry and serum assays in patients with thrombosis and sepsis. Int J Lab Hematol. 2018;40 (4):392-399. doi:10.1111/ijlh.12800

27. Brinkmann V, Reichard U, Goosmann C, et al. Neutrophil extracellular traps kill bacteria. Science. 2004;303(5663):1532-1535. doi:10.1126/science. 1092385

28. Angus DC, Linde-Zwirble WT, Lidicker J, Clermont G, Carcillo J, Pinsky MR. Epidemiology of severe sepsis in the United States: analysis of incidence, outcome, and associated costs of care. Crit Care Med. 2001;29(7):1303-1310. doi:10.1097/00003246200107000-00002

29. Carlet J, Cohen J, Calandra T, Opal SM, Masur H. Sepsis: time to reconsider the concept. Crit Care Med. 2008;36(3):964-966. doi:10.1097/CCM.0B013E318165B886

30. Riedemann NC, Guo RF, Ward PA. The enigma of sepsis. $J$ Clin Invest. 2003;112(4):460-467. doi:10.1172/JCI200319523

31. Pilecky M, Schildberger A, Orth-Holler D, Weber V. Pathogen enrichment from human whole blood for the diagnosis of bloodstream infection: prospects and limitations. Diagn Microbiol Infect Dis. 2019;94(1):7-14. doi:10.1016/j.diagmicrobio.2018.11.015
32. Gyawali B, Ramakrishna K, Dhamoon AS. Sepsis: the evolution in definition, pathophysiology, and management. SAGE Open Med. 2019;7:2050312119835043. doi:10.1177/2050312119835043

33. Horak J, Martinkova V, Radej J, Matejovic M. Back to basics: recognition of sepsis with new definition. J Clin Med. 2019;8:11. doi: $10.3390 / \mathrm{jcm} 8111838$

34. Galley HF, Davies MJ, Webster NR. Ascorbyl radical formation in patients with sepsis: effect of ascorbate loading. Free Radic Biol Med. 1996;20(1):139-143. doi:10.1016/0891-5849(95)02022-5

35. Spoelstra-de Man AME, Elbers PWG, Oudemans-van Straaten HM, Vitamin C. Should we supplement? Curr Opin Crit Care. 2018;24 (4):248-255. doi:10.1097/MCC.0000000000000510

36. Carr AC, Rosengrave PC, Bayer S, Chambers S, Mehrtens J, Shaw GM. Hypovitaminosis $\mathrm{C}$ and vitamin $\mathrm{C}$ deficiency in critically ill patients despite recommended enteral and parenteral intakes. Crit Care. 2017;21(1):300. doi:10.1186/s13054-017-1891-y

37. Long CL, Maull KI, Krishnan RS, et al. Ascorbic acid dynamics in the seriously ill and injured. J Surg Res. 2003;109(2):144-148. doi:10.1016/S0022-4804(02)00083-5

38. Lerman YV, Kim M. Neutrophil migration under normal and sepsis conditions. Cardiovasc Hematol Disord Drug Targets. 2015;15 (1):19-28. doi:10.2174/1871529X15666150108113236

39. Arraes SM, Freitas MS, da Silva SV, et al. Impaired neutrophil chemotaxis in sepsis associates with GRK expression and inhibition of actin assembly and tyrosine phosphorylation. Blood. 2006;108 (9):2906-2913. doi:10.1182/blood-2006-05-024638

40. Alves-Filho JC, de Freitas A, Russo M, Cunha FQ. Toll-like receptor 4 signaling leads to neutrophil migration impairment in polymicrobial sepsis. Crit Care Med. 2006;34(2):461-470. doi:10.1097/01. CCM.0000198527.71819.E1

41. Taneja R, Sharma AP, Hallett MB, Findlay GP, Morris MR. Immature circulating neutrophils in sepsis have impaired phagocytosis and calcium signaling. Shock. 2008;30(6):618-622. doi:10.1097/ SHK.0b013e318173ef9c

42. Wright HL, Moots RJ, Edwards SW. The multifactorial role of neutrophils in rheumatoid arthritis. Nat Rev Rheumatol. 2014;10 (10):593-601. doi:10.1038/nrrheum.2014.80

43. Taneja R, Parodo J, Jia SH, Kapus A, Rotstein OD, Marshall JC. Delayed neutrophil apoptosis in sepsis is associated with maintenance of mitochondrial transmembrane potential and reduced caspase-9 activity. Crit Care Med. 2004;32(7):1460-1469. doi:10.1097/01. CCM.0000129975.26905.77

44. Keel M, Ungethum U, Steckholzer U, et al. Interleukin-10 counterregulates proinflammatory cytokine-induced inhibition of neutrophil apoptosis during severe sepsis. Blood. 1997;90(9):3356-3363. doi:10.1182/blood.V90.9.3356

45. Chapman EA, Lyon M, Simpson D, et al. Caught in a trap? Proteomic analysis of neutrophil extracellular traps in rheumatoid arthritis and systemic lupus erythematosus. Front Immunol. 2019;10:423. doi:10.3389/fimmu.2019.00423

46. O'Brien XM, Biron BM, Reichner JS. Consequences of extracellular trap formation in sepsis. Curr Opin Hematol. 2017;24(1):66-71. doi:10.1097/MOH.0000000000000303

47. Li RHL, Tablin F. A comparative review of neutrophil extracellular traps in sepsis. Front Vet Sci. 2018;5:291. doi:10.3389/fvets.2018.00291

48. Gavillet M, Martinod K, Renella R, et al. Flow cytometric assay for direct quantification of neutrophil extracellular traps in blood samples. Am J Hematol. 2015;90(12):1155-1158. doi:10.1002/ajh.24185

49. Rohrbach AS, Slade DJ, Thompson PR, Mowen KA. Activation of PAD4 in NET formation. Front Immunol. 2012;3:360. doi:10.3389/ fimmu. 2012.00360

50. Carr AC, Maggini S. Vitamin C and Immune Function. Nutrients. 2017;9(11). doi:10.3390/nu9111211

51. Goldschmidt MC, Masin WJ, Brown LR, Wyde PR. The effect of ascorbic acid deficiency on leukocyte phagocytosis and killing of actinomyces viscosus. Int J Vitam Nutr Res. 1988;58(3):326-334. 
52. Parker WH, Rhea EM, Qu ZC, Hecker MR, May JM. Intracellular ascorbate tightens the endothelial permeability barrier through Epac1 and the tubulin cytoskeleton. Am J Physiol Cell Physiol. 2016;311(4): C652-C662. doi:10.1152/ajpcell.00076.2016

53. Ferron-Celma I, Mansilla A, Hassan L, et al. Effect of vitamin $\mathrm{C}$ administration on neutrophil apoptosis in septic patients after abdominal surgery. J Surg Res. 2009;153(2):224-230. doi:10.1016/j. jss.2008.04.024

54. Fisher BJ, Seropian IM, Kraskauskas D, et al. Ascorbic acid attenuates lipopolysaccharide-induced acute lung injury. Crit Care Med. 2011;39(6):1454-1460. doi:10.1097/CCM.0b013e3182120cb8

55. Fisher BJ, Kraskauskas D, Martin EJ, et al. Attenuation of sepsis-induced organ injury in mice by vitamin C. JPEN J Parenter Enteral Nutr. 2014;38(7):825-839. doi:10.1177/0148607113497760
56. Liugan M, Carr AC. Vitamin C and neutrophil function: findings from randomized controlled trials. Nutrients. 2019;11(9):9. doi:10.3390/nu11092102

57. Koba M, Szostek A, Konopa J. Limitation of usage of PicoGreen dye in quantitative assays of double-stranded DNA in the presence of intercalating compounds. Acta Biochim Pol. 2007;54(4):883-886. doi:10.18388/abp.2007 3193

58. Langereis JD. Neutrophil integrin affinity regulation in adhesion, migration, and bacterial clearance. Cell Adh Migr. 2013;7 (6):476-481. doi:10.4161/cam.27293

\section{Publish your work in this journal}

The Journal of Inflammation Research is an international, peerreviewed open-access journal that welcomes laboratory and clinical findings on the molecular basis, cell biology and pharmacology of inflammation including original research, reviews, symposium reports, hypothesis formation and commentaries on: acute/chronic inflammation; mediators of inflammation; cellular processes; molecular mechanisms; pharmacology and novel anti-inflammatory drugs; clinical conditions involving inflammation. The manuscript management system is completely online and includes a very quick and fair peerreview system. Visit http://www.dovepress.com/testimonials.php to read real quotes from published authors. 